\title{
Article \\ Cooling under Applied Stress Rejuvenates Amorphous Alloys and Enhances Their Ductility
}

Nikolai V. Priezjev ${ }^{1,2}$ D

1 Department of Mechanical and Materials Engineering, Wright State University, Dayton, OH 45435, USA; nikolai.priezjev@wright.edu

2 National Research University Higher School of Economics, Myasnitskaya 20, 101000 Moscow, Russia

\begin{abstract}
The effect of tensile stress applied during cooling of binary glasses on the potential energy states and mechanical properties is investigated using molecular dynamics simulations. We study the three-dimensional binary mixture that was first annealed near the glass transition temperature and then rapidly cooled under tension into the glass phase. It is found that at larger values of applied stress, the liquid glass former freezes under higher strain and its potential energy is enhanced. For a fixed cooling rate, the maximum tensile stress that can be applied during cooling is reduced upon increasing initial temperature above the glass transition point. We also show that the amorphous structure of rejuvenated glasses is characterized by an increase in the number of contacts between smaller type atoms. Furthermore, the results of tensile tests demonstrate that the elastic modulus and the peak value of the stress overshoot are reduced in glasses prepared at larger applied stresses and higher initial temperatures, thus indicating enhanced ductility. These findings might be useful for the development of processing and fabrication methods to improve plasticity of bulk metallic glasses.
\end{abstract}

Keywords: metallic glasses; glass transition; rejuvenation; thermomechanical processing; yield stress; molecular dynamics simulations

check for

updates

Citation: Priezjev, N.V. Cooling under Applied Stress Rejuvenates Amorphous Alloys and Enhances Their Ductility. Metals 2021, 11, 67. https://dx.doi.org/10.3390/met11 010067

Received: 8 December 2020 Accepted: 27 December 2020 Published: 31 December 2020

Publisher's Note: MDPI stays neutral with regard to jurisdictional clai$\mathrm{ms}$ in published maps and institutional affiliations.

Copyright: $\odot 2020$ by the authors. Licensee MDPI, Basel, Switzerland. This article is an open access article distributed under the terms and conditions of the Creative Commons Attribution (CC BY) license (https:// creativecommons.org/licenses/by/ $4.0 /)$.

\section{Introduction}

Understanding the relation between amorphous structure and mechanical and physical properties of disordered solids is important for numerous biomedical and structural applications [1,2]. Bulk metallic glasses are known to posses advantageous properties, including high yield stress and large elastic limit, but they become brittle when in a relaxed state [3-8]. To increase ductility, metallic glasses can be rejuvenated via thermal treatment or mechanical agitation [9]. In general, the processing methods that are used to control the energy states of amorphous alloys can be broadly distinguished in two categories based on their temperature relative to $T_{g}$. The first one deals with either cooling from the liquid state into the glass phase or flash annealing, where a glass is temporarily brought to the liquid state and then cooled below $T_{g}$ with a suitably fast rate [10-13]. Other methods involve mechanical and thermal manipulation of amorphous materials within the glass phase. Common examples include ion irradiation [14,15], high-pressure torsion [16,17], shot peening [18,19], elastostatic loading [20-29], and thermal cycling [30-38], among others. On the other hand, amorphous alloys can be mechanically annealed to lower energy states by applying time periodic deformation with an amplitude below the yielding point [39-43], and the decay of the potential energy is accelerated when the loading orientation is periodically alternated in two or three spatial dimensions [44,45]. Most recently, it was discovered experimentally that upon cooling under tension, bulk metallic glass formers become highly rejuvenated and their bending ductility can be tripled [46]. These findings were rationalized by invoking the concept of fictive temperature upon cooling when the characteristic timescale of structural relaxation is comparable to the inverse cooling rate. Moreover, the fictive temperature can be increased by the application of sufficiently high strain rate during freezing, which leads to enhanced ductility [46]. However, despite recent progress, 
optimization and design of the cooling protocols, including stress- versus strain-controlled deformation as well as the range of annealing temperatures and applied stresses, remain a challenging problem.

In this paper, the influence of initial temperature and tensile stress applied during the cooling process on rejuvenation and mechanical properties of binary glasses is studied via molecular dynamics simulations. We consider a binary mixture initially annealed at temperatures slightly above the glass transition temperature and then rapidly cooled under applied tensile stress deep into the glass phase. It will be shown that with increasing tensile stress, glasses are relocated to higher energy states and become more ductile. Moreover, for a given cooling rate, the initial temperature determines the maximum value of the applied stresses and therefore the maximum strain rate during freezing.

The rest of the paper proceeds as follows. The parameters of the interaction potential and the preparation procedure as well as the protocol for cooling under stress are described in the next section. The results for the system deformation, potential energy, atomic structure, and mechanical properties are presented in Section 3. The main conclusions are briefly summarized in the last section.

\section{Molecular Dynamics Simulations}

In our study, the model glass is represented via a mixture of two types of atoms with strongly non-additive interaction that prevents crystallization when the system is cooled below the glass transition temperature [47]. This model was first introduced by Kob and Andersen (KA) about twenty years ago and has since been extensively studied in the context of glass transition, dynamical heterogeneity, and yielding in amorphous materials [47]. It should be noted that the parameters of the KA binary mixture model are similar to those used by Weber and Stillinger to study the amorphous metal alloy $\mathrm{Ni}_{80} \mathrm{P}_{20}$ [48]. In the KA model, the interaction between atoms of types $\alpha, \beta=A, B$ is described by the Lennard-Jones (LJ) potential as follows,

$$
V_{\alpha \beta}(r)=4 \varepsilon_{\alpha \beta}\left[\left(\frac{\sigma_{\alpha \beta}}{r}\right)^{12}-\left(\frac{\sigma_{\alpha \beta}}{r}\right)^{6}\right],
$$

where $\varepsilon_{A A}=1.0, \varepsilon_{A B}=1.5, \varepsilon_{B B}=0.5, \sigma_{A A}=1.0, \sigma_{A B}=0.8, \sigma_{B B}=0.88$, and $m_{A}=m_{B}$ [47]. The total number of atoms is $N=60,000$. To alleviate the computational burden, the cut-off radius of the LJ potential was fixed to $r_{c, \alpha \beta}=2.5 \sigma_{\alpha \beta}$. The results are reported in terms of the LJ units of length, mass, energy, and time, as follows, $\sigma=\sigma_{A A}$, $m=m_{A}, \varepsilon=\varepsilon_{A A}$, and, correspondingly, $\tau=\sigma \sqrt{m / \varepsilon}$. The MD simulations were performed in parallel using the efficient LAMMPS code [49] with the integration time step $\triangle t_{M D}=0.005 \tau$, periodic boundary conditions, and the Nosé-Hoover thermostat [50].

The first step in the preparation procedure was to equilibrate the binary mixture near the glass transition temperature at zero pressure. It was originally demonstrated, by extrapolating diffusion coefficients of both types of atoms as a function of temperature, that the critical temperature of the KA model at the constant density $\rho=\rho_{A}+\rho_{B}=1.2 \sigma^{-3}$ is $T_{\mathcal{C}}=0.435 \varepsilon / k_{B}$ [47]. More recently, it was found from the crossover of the potential energy curve that the glass transition temperature is about $T_{g} \approx 0.35 \varepsilon / k_{B}$ when the system is cooled from the liquid state with the rate $10^{-5} \varepsilon / k_{B} \tau$ at zero pressure [12]. In the present study, the binary mixture was equilibrated at $T_{L J}=1.0 \varepsilon / k_{B}$ and then annealed at four temperatures near $T_{g}$, namely, $T_{L J}=0.36 \varepsilon / k_{B}, 0.38 \varepsilon / k_{B}, 0.40 \varepsilon / k_{B}$, and $0.42 \varepsilon / k_{B}$, during $2 \times 10^{5} \tau$ in a periodic box at $P=0$. It was previously shown that the binary mixture undergoes structural relaxation during the time interval $2 \times 10^{5} \tau$ at $T_{L J} \geqslant 0.32 \varepsilon / k_{B}$, and the potential energy of inherent structures decreases upon reducing annealing temperature towards $T_{g}$ [6].

Following the equilibration period, the system was linearly cooled from the initial temperatures $\left(0.36 \varepsilon / k_{B}, 0.38 \varepsilon / k_{B}, 0.40 \varepsilon / k_{B}\right.$, and $\left.0.42 \varepsilon / k_{B}\right)$ to $T_{L J}=0.01 \varepsilon / k_{B}$ at a rate of $10^{-4} \varepsilon / k_{B} \tau$. In addition, a constant stress along the $\hat{z}$ direction was applied during the cooling process, while the normal stress components along the other two directions 
were set to zero (see Figure 1). For reference, the simulations of the cooling process at zero pressure along all three directions were also carried out. After cooling, the applied stress was set to zero and the glass was allowed to equilibrate during the additional time interval of $10^{4} \tau$ at $T_{L J}=0.01 \varepsilon / k_{B}$ and $P=0$. Finally, the glassy samples were strained at the constant rate of $10^{-5} \tau^{-1}$ at $T_{L J}=0.01 \varepsilon / k_{B}$ and zero pressure in order to evaluate the elastic modulus and the peak value of the stress overshoot. The data for the potential energy and mechanical properties were averaged over 15 independent samples.

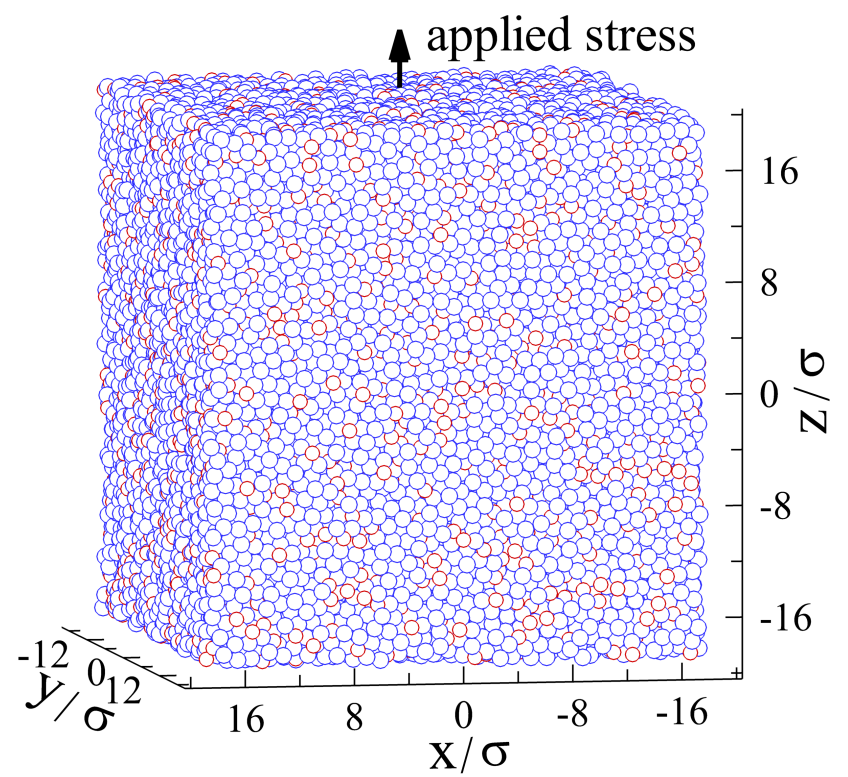

Figure 1. (Color online) The snapshot of the binary glass after cooling from $T_{L J}=0.36 \varepsilon / k_{B}$ to $0.01 \varepsilon / k_{B}$ with the rate $10^{-4} \varepsilon / k_{B} \tau$ and at the same time applying the constant stress $0.6 \varepsilon \sigma^{-3}$ along the $\hat{z}$ direction. The stress components along the $\hat{x}$ and $\hat{y}$ directions are set to zero. The total number of atoms is 60,000 . The atoms of types $A$ and $B$ are denoted by large blue and small red spheres, respectively. The atoms are not drawn to scale.

\section{Results}

It is now well understood that the potential energy of glasses and, consequently, their mechanical properties depend sensitively on the rate of cooling from the liquid state [9]. In general, more slowly cooled glass formers settle at lower energy states, and, under external deformation, exhibit higher yield stress and are more brittle [51]. Remarkably, it was recently demonstrated experimentally that ductility can be enhanced when sufficiently large normal stress is applied during the cooling process, leading to highly rejuvenated states [46]. The key parameters of the cooling under stress protocol include the initial temperature of a glass former, the rate of cooling, and the applied stress. In the present study, the binary mixture was cooled under stress with the fixed rate of $10^{-4} \varepsilon / k_{B} \tau$. We note that cooling at higher rates, $10^{-2} \varepsilon / k_{B} \tau$ and $10^{-3} \varepsilon / k_{B} \tau$, and zero pressure was shown to produce highly rejuvenated glassy samples with already low values of the yielding peak [32,34]. On the other hand, test runs at the lower cooling rate, $10^{-5} \varepsilon / k_{B} \tau$, did not reveal significant rejuvenation, in part because only a relatively small stresses can be applied during the cooling process without extended flow near the glass transition temperature. Therefore, for each initial temperature near $T_{g}$, the values of the applied stress were chosen by trial and error up to the maximum stress above which samples break.

In our setup, the binary mixture is initially equilibrated near $T_{g}$ in a cubic box with periodic boundaries, and then cooled under stress applied along the $\hat{z}$ direction, which might result in significant deformation of the computational domain. The time dependence of the system size along the $\hat{z}$ direction is reported in Figure 2 for the selected values of the applied stress. For each value of the initial temperature, the variation of $L_{z}$ is shown for 
zero, intermediate value, and the maximum applied stress above which samples undergo extensive plastic deformation near the glass transition temperature. It can be clearly seen in Figure 2 that with increasing applied stress, the deformation becomes more pronounced in the vicinity of $T_{g}=0.35 \varepsilon / k_{B}$, which is marked by the vertical dashed lines. Note that the negative slope at later times corresponds to contraction upon cooling below the glass transition temperature, where the applied stress becomes smaller than the yield stress. Shown for reference in all panels in Figure 2, the black curves denote the variation of $L_{z}$ during the cooling process at zero pressure for different initial temperatures. As deduced from the blue curve in Figure 2c, the maximum elongation upon cooling to $T_{L J}=0.01 \varepsilon / k_{B}$ under stress is $L_{z} / L_{x} \approx 1.6$.

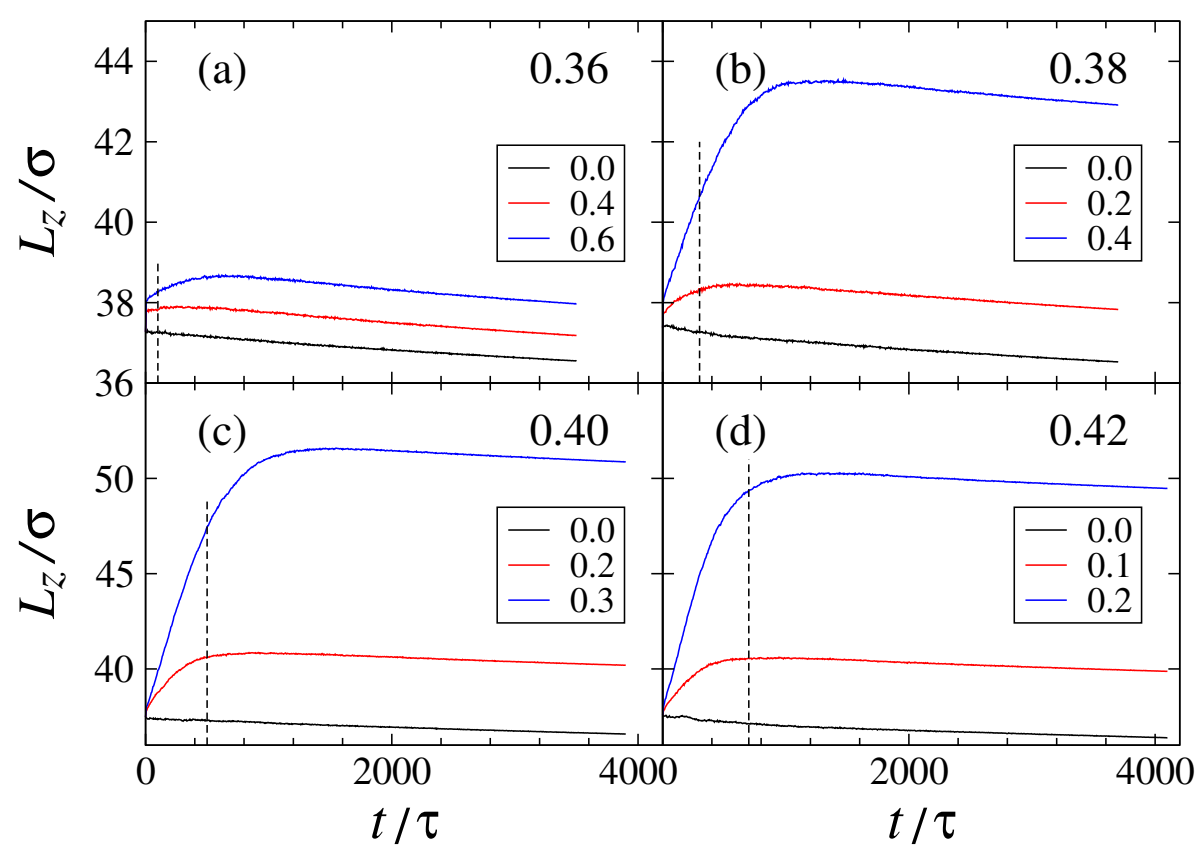

Figure 2. (Color online) The system size along the $\hat{z}$ direction during cooling from the indicated initial temperatures to $T_{L J}=0.01 \varepsilon / k_{B}$ with the rate $10^{-4} \varepsilon / k_{B} \tau$. The initial temperatures are (a) $0.36 \varepsilon / k_{B}$, (b) $0.38 \varepsilon / k_{B}$, (c) $0.40 \varepsilon / k_{B}$, and (d) $0.42 \varepsilon / k_{B}$. The values of the applied stress (in units of $\left.\varepsilon \sigma^{-3}\right)$ are listed in the legend. The vertical dashed lines denote times when the system temperature is $0.35 \varepsilon / k_{B}$. The scales along the vertical axes are different on the upper and lower panels.

The rejuvenated structure in a strained sample is formed mostly during the time interval when the system temperature is reduced in a close vicinity of the glass transition temperature. Otherwise, a liquid glass former flows above $T_{g}$, whereas in the glass phase, the applied stress remains smaller than the yield stress, and the effect of elastostatic loading on the energy state during $\sim 10^{3} \tau$ is negligible [28]. Similar to the definition used in [46], the strain along the $\hat{z}$ direction was computed as

$$
\varepsilon(t)=\left[L_{z}(t)-L_{z}(0)\right] / L_{z}(0) \cdot 100,
$$

where $L_{z}(0)$ is the initial length of the computational domain and $L_{z}(t)$ is the time dependent length. The rate of strain as a function of time is plotted in Figure 3 for the same values of the applied stress and initial temperature as in Figure 2. It can be readily observed that the rate of strain at $T_{g}=0.35 \varepsilon / k_{B}$ is larger at higher initial temperatures and the maximum applied stress (marked by the blue curves in Figure 3 ). The exception to this trend is the case of the initial temperature $T_{L J}=0.42 \varepsilon / k_{B}$ where the maximum applied stress is relatively low, $0.2 \varepsilon \sigma^{-3}$, and most of the deformation occurs just above $T_{g}$ (see Figure 3d). Further, it was argued that the effect of rejuvenation is enhanced when the typical timescale associated with the cooling process (i.e., the inverse cooling rate) exceeds the characteristic time of the straining process, which is roughly a few percent divided by 
the strain rate [46]. For the parameters of the present study, this condition is satisfied for all values of the applied stress, thus leading to rejuvenated states with respect to energy levels obtained upon cooling at $P=0$.

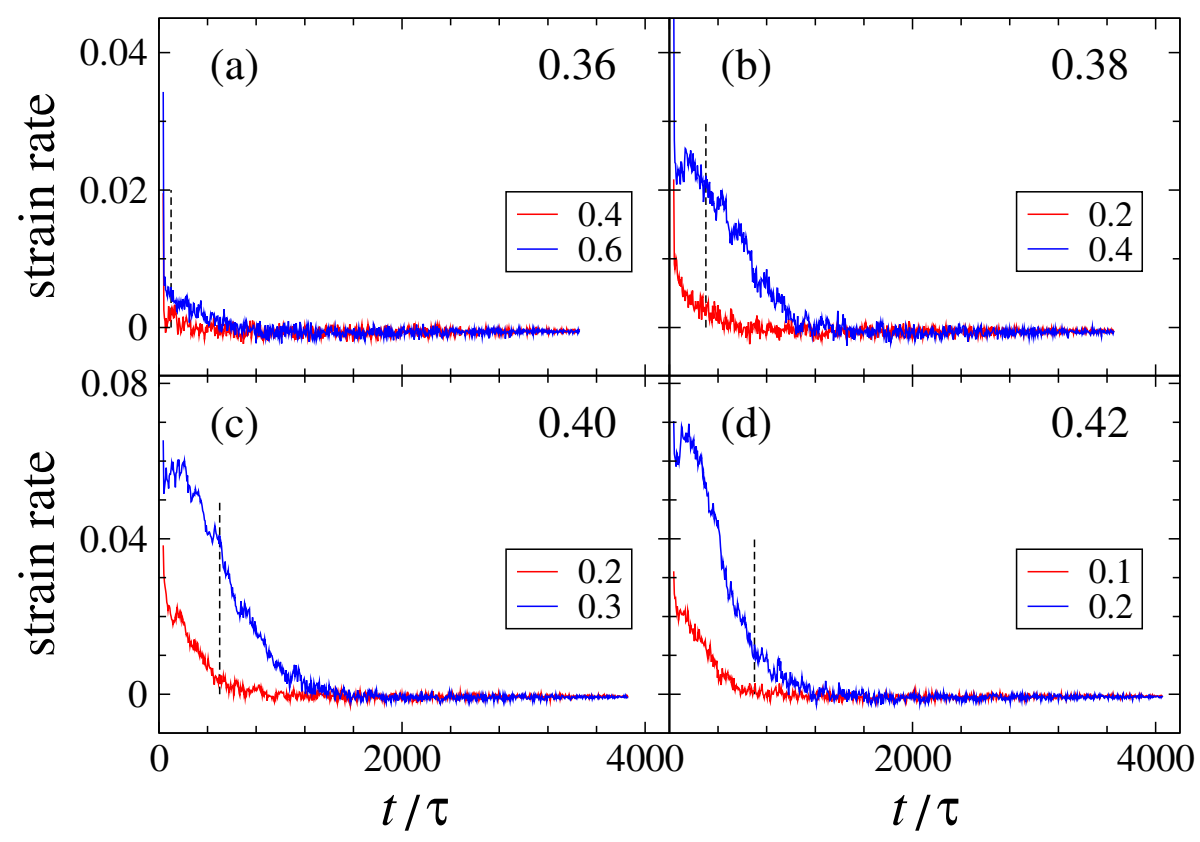

Figure 3. (Color online) The rate of strain along the $\hat{z}$ direction (in units of $\tau^{-1}$ ) during cooling from the initial temperatures to $T_{L J}=0.01 \varepsilon / k_{B}$ with the rate $10^{-4} \varepsilon / k_{B} \tau$. The same data as in Figure 2 . The initial temperatures are (a) $0.36 \varepsilon / k_{B}$, (b) $0.38 \varepsilon / k_{B}$, (c) $0.40 \varepsilon / k_{B}$, and (d) $0.42 \varepsilon / k_{B}$. The applied stress (in units of $\varepsilon \sigma^{-3}$ ) along the $\hat{z}$ direction is indicated in the legend. The vertical dashed lines denote the temperature of $0.35 \varepsilon / k_{B}$. Note that the vertical scales are different on the upper and lower panels.

Next, the variation of the potential energy per atom as a function of stress applied during the cooling process is shown in Figure 4 for the initial temperatures $T_{L J}=0.36 \varepsilon / k_{B}$, $0.38 \varepsilon / k_{B}, 0.40 \varepsilon / k_{B}$, and $0.42 \varepsilon / k_{B}$. The data in Figure 4 were averaged over 15 samples after annealing during $10^{4} \tau$ at $T_{L J}=0.01 \varepsilon / k_{B}$ and $P=0$. It can be seen that for zero applied stress, the potential energy is reduced upon decreasing initial temperature. It was previously demonstrated that the potential energy of the inherent structures of the KA mixture at zero pressure is reduced when the annealing temperature approaches $T_{g}$ from above [6]. Further, with increasing applied stress, the binary glass is relocated to progressively higher energy states as it freezes at higher strain. It should be commented that the maximum increase in the potential energy at $T_{L J}=0.36 \varepsilon / k_{B}$ and $0.38 \varepsilon / k_{B}$ is slightly larger than the most pronounced rejuvenation due to elastostatic loading of the KA glass detected at about half the glass transition temperature [28,29]. On the other hand, it was shown for the same binary mixture model that rejuvenation is significantly enhanced as a result of flush annealing when samples are rapidly heated above $T_{g}$ and cooled at effectively high rates into the glass phase [12]. 


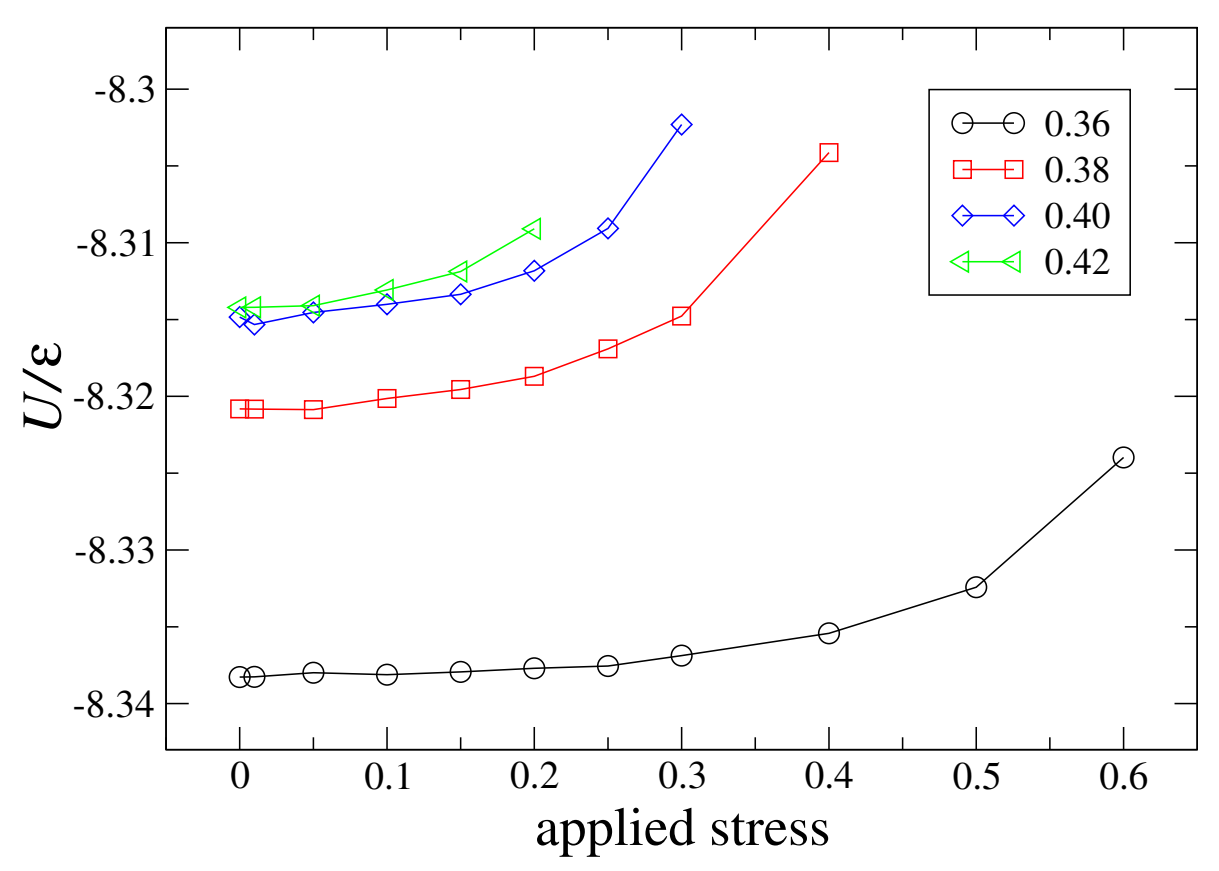

Figure 4. (Color online) The potential energy per atom as a function of the applied stress (in units of $\varepsilon \sigma^{-3}$ ) during cooling to the temperature $T_{L J}=0.01 \varepsilon / k_{B}$ with the rate $10^{-4} \varepsilon / k_{B} \tau$. The values of the initial temperature (in units of $\varepsilon / k_{B}$ ) are listed in the legend. The potential energy is computed at $T_{L J}=0.01 \varepsilon / k_{B}$ and zero pressure. The data were averaged over 15 independent samples.

The increase in potential energy reported in Figure 4 is reflected in the atomic structure, which can be analyzed using the radial distribution function. Thus, it was previously found that the most sensitive measure of the structural changes in the KA model glass is the average separation between neighboring atoms of type $B[6,52,53]$. This correlation can be understood by realizing that the interaction energy between smaller atoms of type $B$ in the LJ potential, Equation (1), is the lowest among $\varepsilon_{A A}, \varepsilon_{A B}$, and $\varepsilon_{B B}$, leading to a reduced number of contacts between $B$ type atoms in a well-annealed (low-energy) glass $[52,53]$. The averaged radial distribution function, $g(r)_{B B}$, is shown in Figure 5 for zero and maximum applied stress during cooling from the indicated initial temperatures to $T_{L J}=0.01 \varepsilon / k_{B}$. It can be observed that upon increasing stress, the height of the first peak at about $0.96 \sigma$ becomes slightly larger and the magnitude of the second peak at $\approx 1.38 \sigma$ is reduced. This trend is consistent with the increase in potential energy when samples are cooled under stress, thus leading to a more random packing and larger number of contacts between $B-B$ type atoms.

In order to evaluate changes in mechanical properties due to cooling under applied stress, the glassy samples were strained at the constant rate of $10^{-5} \tau^{-1}$ at $T_{L J}=0.01 \varepsilon / k_{B}$ and zero pressure. The representative stress-strain curves are plotted in Figure 6 for the indicated values of the initial temperature. In each case, the data are reported for zero and maximum applied stress during the cooling process. As is evident, the height of the yielding peak increases in samples prepared at lower initial temperatures and zero external stress. This is consistent with the results in Figure 4 where more relaxed states were obtained by cooling at zero applied stress and lower temperatures. Moreover, it is clearly seen that the yielding peak in Figure 6 is reduced in highly rejuvenated samples initially cooled at the maximum applied stress. Therefore, it can be concluded that the maximum difference in the yield stress becomes more pronounced for binary glasses prepared at lower initial temperatures. 


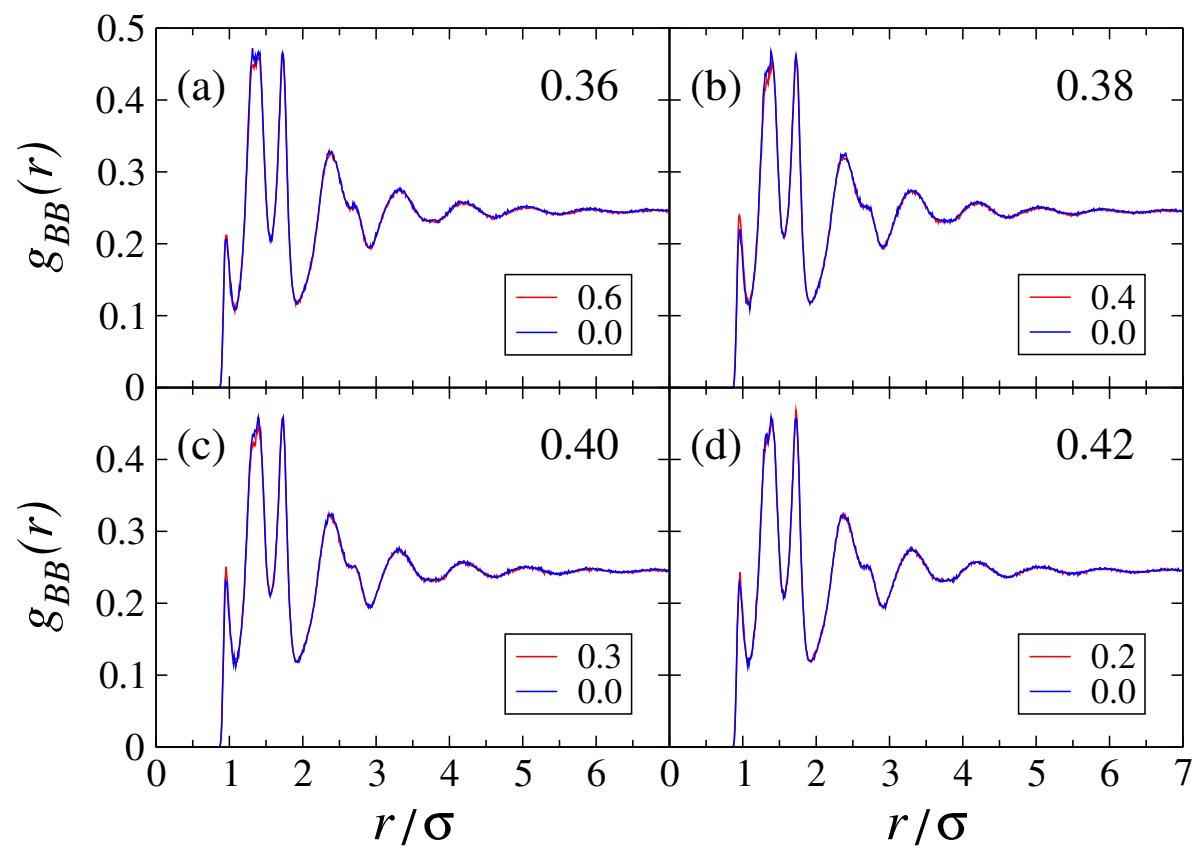

Figure 5. (Color online) The radial distribution function, $g_{B B}(r)$, after cooling to $T_{L J}=0.01 \varepsilon / k_{B}$ from the initial temperatures (a) $0.36 \varepsilon / k_{B}$, (b) $0.38 \varepsilon / k_{B}$, (c) $0.40 \varepsilon / k_{B}$, and (d) $0.42 \varepsilon / k_{B}$. The values of the applied stress (in units of $\varepsilon \sigma^{-3}$ ) along the $\hat{z}$ direction are listed in the legend.

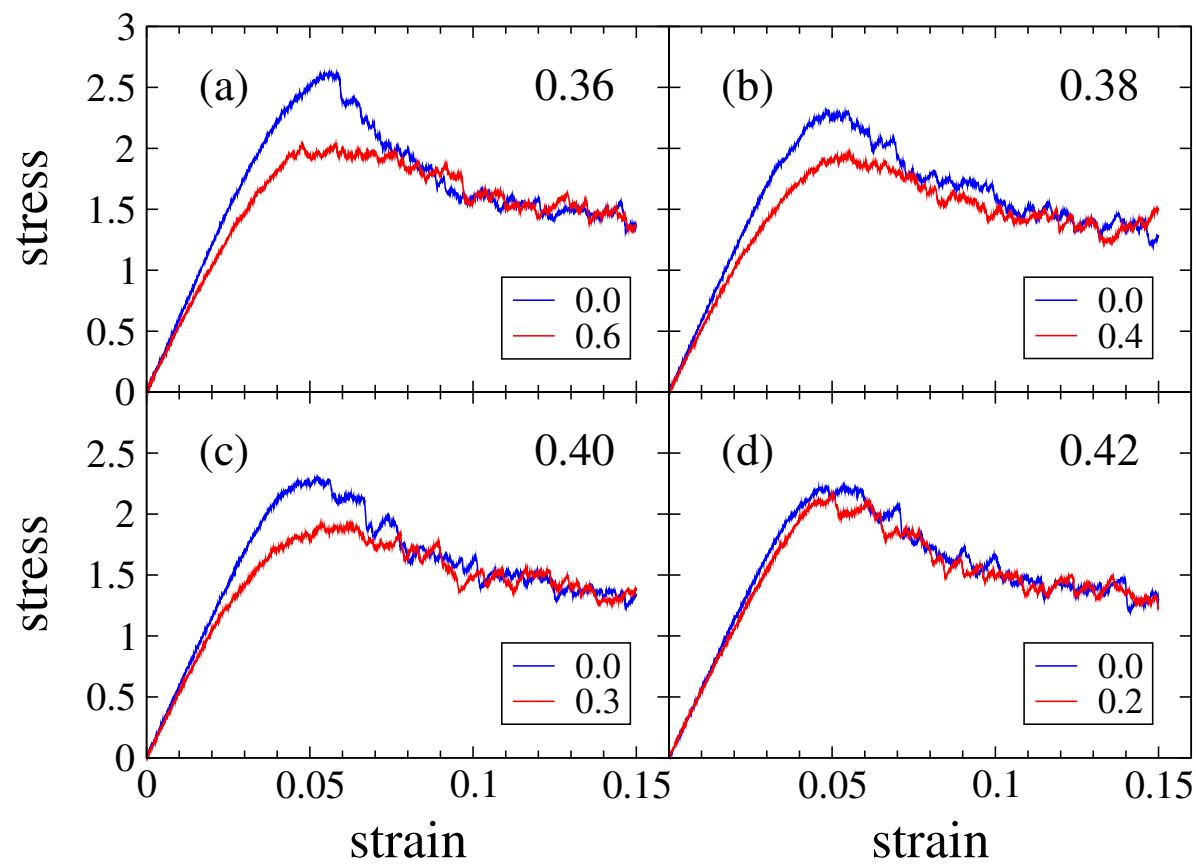

Figure 6. (Color online) The variation of the normal stress (in units of $\varepsilon \sigma^{-3}$ ) as a function of strain along the $\hat{z}$ direction during steady loading with the rate $10^{-5} \tau^{-1}$ at $T_{L J}=0.01 \varepsilon / k_{B}$ and zero pressure. The samples were prepared by cooling from the initial temperatures (a) $0.36 \varepsilon / k_{B}$, (b) $0.38 \varepsilon / k_{B}$, (c) $0.40 \varepsilon / k_{B}$, and (d) $0.42 \varepsilon / k_{B}$. The values of the applied stress during the cooling process are listed in the insets. See text for details.

The simulation results for the elastic modulus, $E$, and the peak value of the stress overshoot, $\sigma_{Y}$, in steadily strained samples at $T_{L J}=0.01 \varepsilon / k_{B}$ are presented in Figures 7 and 8 , respectively. Here, the data were averaged over 15 realizations of disorder. For each sample, the elastic modulus was computed from the best linear fit to stress at small values of strain, $\varepsilon_{z z} \leqslant 0.01$, and the yielding peak represents the maximum of the stress-strain curves in 
the range $\varepsilon_{z z} \leqslant 0.15$. As expected, both $E$ and $\sigma_{Y}$ tend to increase in better-annealed samples that were cooled from lower initial temperatures at zero stress (see Figures 7 and 8). Except for the case $T_{L J}=0.42 \varepsilon / k_{B}$, the elastic modulus is reduced by about $10 \%$ and the yielding peak is decreased by roughly $15 \%$, when the applied stress varies from zero to a maximum value. All in all, these results demonstrate that the process of cooling from initial temperatures near the glass transition temperature and concomitantly applying a constant stress in tension leads to improved plasticity in amorphous alloys.

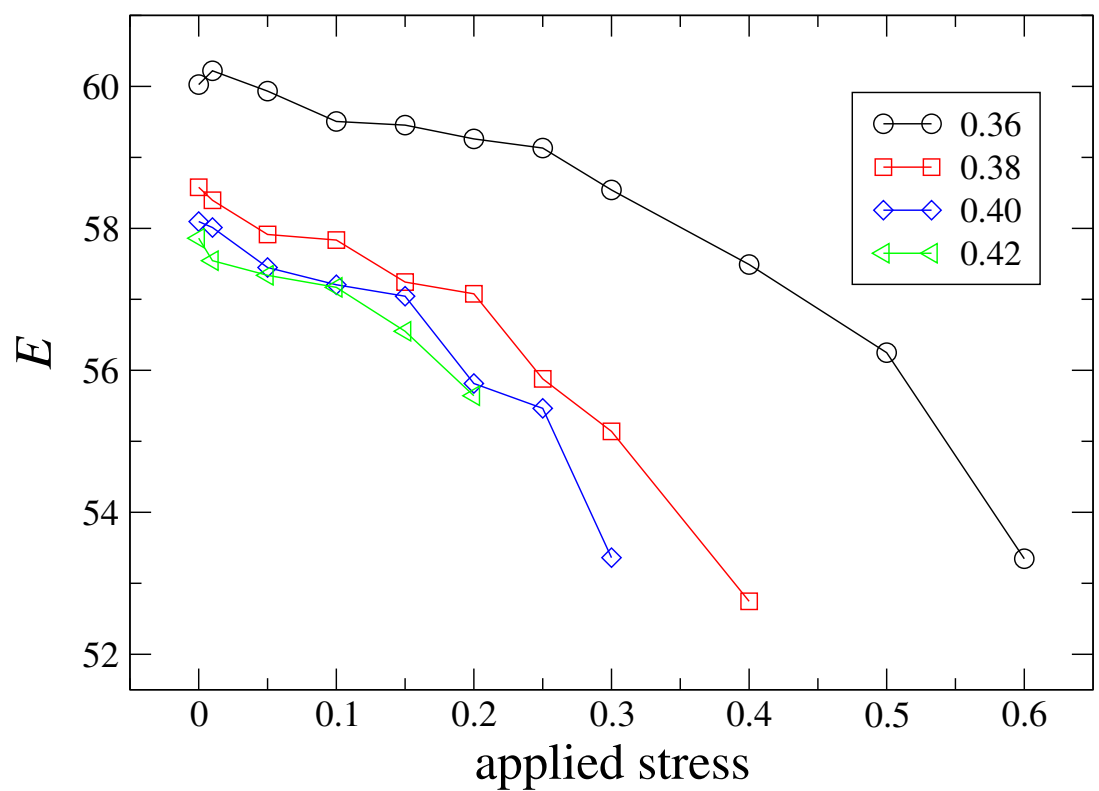

Figure 7. (Color online) The elastic modulus $E$ (in units of $\varepsilon \sigma^{-3}$ ) versus the applied stress (in units of $\varepsilon \sigma^{-3}$ ) used during the cooling process for the tabulated values of the initial temperature. The elastic modulus was measured during tension along the $\hat{z}$ direction with the rate $10^{-5} \tau^{-1}$ at $T_{L J}=0.01 \varepsilon / k_{B}$ and zero pressure.

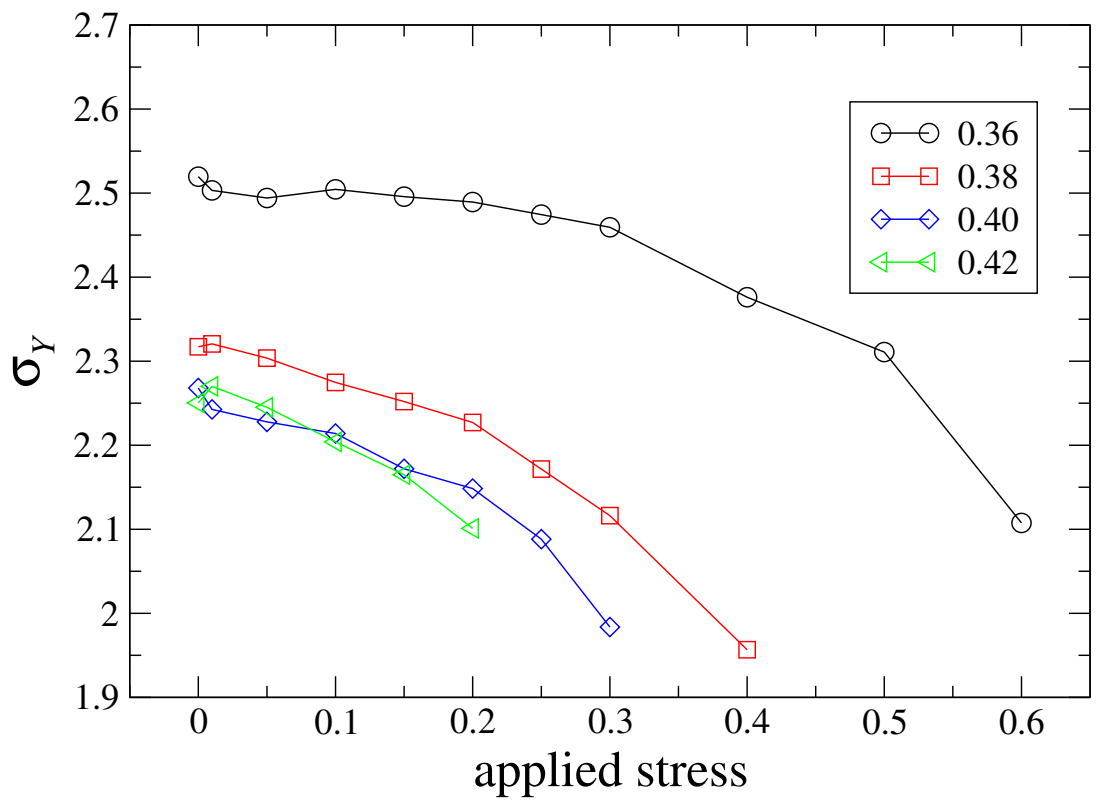

Figure 8. (Color online) The peak value of the stress overshoot $\sigma_{Y}$ (in units of $\varepsilon \sigma^{-3}$ ) as a function the applied stress (in units of $\varepsilon \sigma^{-3}$ ) used during cooling to $T_{L J}=0.01 \varepsilon / k_{B}$ for the indicated values of the initial temperature. The same samples and loading conditions as in Figure 7. 


\section{Conclusions}

In summary, we studied the effect of strain rate applied during the cooling of amorphous alloys on their energy states and mechanical properties using atomistic simulations. The model glass was represented via a binary mixture which was first equilibrated near the glass transition temperature and then linearly cooled under constant tensile stress deep into the glass phase. It was found that the potential energy of binary glasses increases when a larger tensile stress was applied during the cooling process, resulting in a higher strain rate during freezing near the glass transition temperature. In turn, the maximum value of the applied stress is reduced at higher initial temperatures as glassy samples can be extensively deformed above $T_{g}$. Furthermore, it was shown that the amorphous structure of rejuvenated glasses contains a larger number of contacts between smaller atoms, as reflected in the shape of the radial distribution function. Last, the ductility is enhanced as the elastic modulus and the yielding peak are reduced in glasses that were cooled at larger tensile stresses and higher initial temperatures.

Funding: The financial support from the National Science Foundation (CNS-1531923) and the ACS Petroleum Research Fund (60092-ND9) is gratefully acknowledged. The article was prepared within the framework of the HSE University Basic Research Program and funded in part by the Russian Academic Excellence Project "5-100".

Data Availability Statement: The data presented in this study are available upon request.

Acknowledgments: The author would like to thank J. Schroers for useful comments. The molecular dynamics simulations were performed using the LAMMPS open source code developed at Sandia National Laboratories [49]. The numerical simulations were carried out at the Wright State University's Computing Facility and the Ohio Supercomputer Center.

Conflicts of Interest: The author declares that he has no conflict of interest.

\section{References}

1. Khan, M.M.; Nemati, A.; Rahman, Z.U.; Shah, U.H.; Asgar, H.; Haider, W. Recent advancements in bulk metallic glasses and their applications: A Review. Crit. Rev. Solid State Mater. Sci. 2018, 43, 233-268. [CrossRef]

2. Li, H.F.; Zheng, Y.F. Recent advances in bulk metallic glasses for biomedical applications. Acta Biomater. 2016, 36, 1-20. [CrossRef] [PubMed]

3. Schuh, C.A.; Lund, A.C. Atomistic basis for the plastic yield criterion of metallic glass. Nat. Mater. 2003, 2, 449-452. [CrossRef] [PubMed]

4. Ding, J.; Cheng, Y.Q.; Ma, E. Correlating local structure with inhomogeneous elastic deformation in a metallic glass. Appl. Phys. Lett. 2012, 101, 121917. [CrossRef]

5. Shang, B.S.; Li, M.Z.; Yao, Y.G.; Lu, Y.J.; Wang, W.H. Evolution of atomic rearrangements in deformation in metallic glasses. Phys. Rev. E 2014, 90, 042303. [CrossRef]

6. Priezjev, N.V. The effect of thermal history on the atomic structure and mechanical properties of amorphous alloys. Comput. Mater. Sci. 2020, 174, 109477. [CrossRef]

7. Priezjev, N.V. Spatiotemporal analysis of nonaffine displacements in disordered solids sheared across the yielding point. Metall. Mater. Trans. A 2020, 51, 3713. [CrossRef]

8. Singh, M.; Ozawa, M.; Berthier, L. Brittle yielding of amorphous solids at finite shear rates. Phys. Rev. Mater. 2020, 4, 025603. [CrossRef]

9. Sun, Y.; Concustell, A.; Greer, A.L. Thermomechanical processing of metallic glasses: Extending the range of the glassy state. Nat. Rev. Mater. 2016, 1, 16039. [CrossRef]

10. Wakeda, M.; Saida, J.; Li, J.; Ogata, S. Controlled rejuvenation of amorphous metals with thermal processing. Sci. Rep. 2015, 5, 10545. [CrossRef]

11. Kuchemann, S.; Derlet, P.M.; Liu, C.; Rosenthal, D.; Sparks, G.; Larson, W.S.; Maass, R. Energy storage in metallic glasses via flash annealing. Adv. Funct. Mater. 2018, 28, 1805385. [CrossRef]

12. Priezjev, N.V. Atomistic modeling of heat treatment processes for tuning the mechanical properties of disordered solids. J. NonCryst. Solids 2019, 518, 128-133. [CrossRef]

13. Wang, M.; Liu, H.; Li, J.; Jiang, Q.; Yang, W.; Tang, C. Thermal-pressure treatment for tuning the atomic structure of metallic glass Cu-Zr. J. Non-Cryst. Solids 2020, 535, 119963. [CrossRef]

14. Magagnosc, D.J.; Kumar, G.; Schroers, J.; Felfer, P.; Cairney, J.M.; Gianola, D.S. Effect of ion irradiation on tensile ductility, strength and fictive temperature in metallic glass nanowires. Acta Mater. 2014, 74, 165-182. [CrossRef] 
15. Xue, P.; Pauly, S.; Gan, W.; Jiang, S.; Fan, H.; Ning, Z.; Huang, Y.; Sun, J. Enhanced tensile plasticity of a CuZr-based bulk metallic glass composite induced by ion irradiation. J. Mater. Sci. Technol. 2019, 35, 2221-2226. [CrossRef]

16. Meng, F.; Tsuchiya, K.; Li, S.; Yokoyama, Y. Reversible transition of deformation mode by structural rejuvenation and relaxation in bulk metallic glass. Appl. Phys. Lett. 2012, 101, 121914. [CrossRef]

17. Wang, Y.B.; Qu, D.D.; Wang, X.H.; Cao, Y.; Liao, X.Z.; Kawasaki, M.; Ringer, S.P.; Shan, Z.W.; Langdon, T.G.; Shen, J. Introducing a strain-hardening capability to improve the ductility of bulk metallic glasses via severe plastic deformation. Acta Mater. 2012, 60, 253-260. [CrossRef]

18. Mear, F.O.; Vaughan, G.; Yavari, A.R.; Greer, A.L. Residual-stress distribution in shot-peened metallic-glass plate. Philos. Mag. Lett. 2008, 88, 757-766. [CrossRef]

19. Gonzalez, S.; Fornell, J.; Pellicer, E.; Surinach, S.; Baro, M.D.; Greer, A.L.; Belzunce, F.J.; Sort, J. Influence of the shot-peening intensity on the structure and near-surface mechanical properties of $\mathrm{Ti}_{40} \mathrm{Zr}_{10} \mathrm{Cu}_{38} \mathrm{Pd}_{12}$ bulk metallic glass. Appl. Phys. Lett. 2013, 103, 211907. [CrossRef]

20. Park, K.-W.; Lee, C.-M.; Wakeda, M.; Shibutani, Y.; Falk, M.L.; Lee, J.-C. Elastostatically induced structural disordering in amorphous alloys. Acta Mater. 2008, 56, 5440-5450. [CrossRef]

21. Lee, C.-M.; Park, K.-W.; Lee, B.-J.; Shibutani, Y.; Lee, J.-C. Structural disordering of amorphous alloys: A molecular dynamics analysis. Scr. Mater. 2009, 61, 911-914. [CrossRef]

22. Tong, Y.; Dmowski, W.; Yokoyama, Y.; Wang, G.; Liaw, P.K.; Egami, T. Recovering compressive plasticity of bulk metallic glasses by high-temperature creep. Scr. Mater. 2013, 69, 570-573. [CrossRef]

23. Wang, Y.M.; Zhang, M.; Liu, L. Mechanical annealing in the homogeneous deformation of bulk metallic glass under elastostatic compression. Scr. Mater. 2015, 102, 67-70. [CrossRef]

24. Zhao, L.Z.; Xue, R.J.; Li, Y.Z.; Wang, W.H.; Bai, H.Y. Revealing localized plastic flow in apparent elastic region before yielding in metallic glasses. J. Appl. Phys. 2015, 118, 244901. [CrossRef]

25. Greer, A.L.; Sun, Y.H. Stored energy in metallic glasses due to strains within the elastic limit. Philos. Mag. 2016, 96, 1643-1663. [CrossRef]

26. Zhang, M.; Wang, Y.M.; Li, F.X.; Jiang, S.Q.; Li, M.Z.; Liu, L. Mechanical relaxation-to-rejuvenation transition in a Zr-based bulk metallic glass. Sci. Rep. 2017, 7, 625. [CrossRef]

27. Pan, J.; Wang, Y.X.; Guo, Q.; Zhang, D.; Greer, A.L.; Li, Y. Extreme rejuvenation and softening in a bulk metallic glass. Nat. Commun. 2018, 9, 560. [CrossRef]

28. Priezjev, N.V. Aging and rejuvenation during elastostatic loading of amorphous alloys: A molecular dynamics simulation study. Comput. Mater. Sci. 2019, 168, 125-130. [CrossRef]

29. Priezjev, N.V. Accelerated rejuvenation in metallic glasses subjected to elastostatic compression along alternating directions. J. Non-Cryst. Solids 2020, 120562. [CrossRef]

30. Ketov, S.V.; Sun, Y.H.; Nachum, S.; Lu, Z.; Checchi, A.; Beraldin, A.R.; Bai, H.Y.; Wang, W.H.; Louzguine-Luzgin, D.V.; Carpenter, M.A.; et al. Rejuvenation of metallic glasses by non-affine thermal strain. Nature 2015, 524, 200-203. [CrossRef]

31. Guo, W.; Saida, J.; Zhao, M.; Lu, S.; Wu, S. Rejuvenation of Zr-based bulk metallic glass matrix composite upon deep cryogenic cycling. Mater. Lett. 2019, 247, 135-138. [CrossRef]

32. Priezjev, N.V. The effect of cryogenic thermal cycling on aging, rejuvenation, and mechanical properties of metallic glasses. J. Non-Cryst. Solids 2019, 503, 131-138. [CrossRef]

33. Liu, Q.-L.; Priezjev, N.V. The influence of complex thermal treatment on mechanical properties of amorphous materials. Comput. Mater. Sci. 2019, 161, 93-98. [CrossRef]

34. Priezjev, N.V. Potential energy states and mechanical properties of thermally cycled binary glasses. J. Mater. Res. 2019, 34, 2664-2671. [CrossRef]

35. Samavatian, M.; Gholamipour, R.; Amadeh, A.A.; Mirdamadi, S. Correlation between plasticity and atomic structure evolution of a rejuvenated bulk metallic glass. Metall. Mater. Trans. A 2019, 50, 4743-4749. [CrossRef]

36. Ketkaew, J.; Yamada, R.; Wang, H.; Kuldinow, D.; Schroers, B.S.; Dmowski, W.; Egami, T.; Schroers, J. The effect of thermal cycling on the fracture toughness of metallic glasses. Acta Mater. 2020, 184, 100. [CrossRef]

37. Meylan, C.M.; Papparotto, F.; Nachum, S.; Orava, J.; Miglierini, M.; Basykh, V.; Ferenc, J.; Kulik, T.; Greer, A.L. Stimulation of shear-transformation zones in metallic glasses by cryogenic thermal cycling. J. Non-Cryst. Solids 2020, 584, 120299. [CrossRef]

38. Du, Y.; Han, W.; Zhou, Q.; Xu, Y.; Zhai, H.; Bhardwaj, V.; Wang, H. Enhancing the plasticity of a Ti-based bulk metallic glass composite by cryogenic cycling treatments. J. Alloys Compd. 2020, 835, 155247. [CrossRef]

39. Lacks, D.J.; Osborne, M.J. Energy landscape picture of overaging and rejuvenation in a sheared glass. Phys. Rev. Lett. 2004, 93, 255501. [CrossRef]

40. Fiocco, D.; Foffi, G.; Sastry, S. Oscillatory athermal quasistatic deformation of a model glass. Phys. Rev. E 2013, 88, 020301(R). [CrossRef]

41. Priezjev, N.V. Molecular dynamics simulations of the mechanical annealing process in metallic glasses: Effects of strain amplitude and temperature. J. Non-Cryst. Solids 2018, 479, 42-48. [CrossRef]

42. Priezjev, N.V. Slow relaxation dynamics in binary glasses during stress-controlled, tension-compression cyclic loading Comput. Mater. Sci. 2018, 153, 235-240. [CrossRef] 
43. Jana, P.K.; Priezjev, N.V. Structural relaxation in amorphous materials under cyclic tension-compression loading. J. Non-Cryst. Solids 2020, 540, 120098. [CrossRef]

44. Priezjev, N.V. Accelerated relaxation in disordered solids under cyclic loading with alternating shear orientation. J. Non-Cryst. Solids 2019, 525, 119683. [CrossRef]

45. Priezjev, N.V. Alternating shear orientation during cyclic loading facilitates yielding in amorphous materials. J. Mater. Eng. Perform. 2020, 29, 7328-7335. [CrossRef]

46. Mota, R.; Lund, E.; Sohn, S.; Browne, D.; Schroers, J. Pulling metallic glasses ductile. Res. Sq. 2020. [CrossRef]

47. Kob, W.; Andersen, H.C. Testing mode-coupling theory for a supercooled binary Lennard-Jones mixture: The van Hove correlation function. Phys. Rev. E 1995, 51, 4626-4641. [CrossRef]

48. Weber, T.A.; Stillinger, F.H. Local order and structural transitions in amorphous metal-metalloid alloys. Phys. Rev. B 1985, 31, 1954-1963. [CrossRef]

49. Plimpton, S.J. Fast parallel algorithms for short-range molecular dynamics. J. Comp. Phys. 1995, 117, 1-19. [CrossRef]

50. Allen, M.P.; Tildesley, D.J. Computer Simulation of Liquids; Clarendon: Oxford, UK, 1987.

51. Fan, M.; Wang, M.; Zhang, K.; Liu, Y.; Schroers, J.; Shattuck, M.D.; O'Hern, C.S. The effects of cooling rate on particle rearrangement statistics: Rapidly cooled glasses are more ductile and less reversible. Phys. Rev. E 2017, 95, 022611. [CrossRef]

52. Vollmayr, K.; Kob, W.; Binder, K. How do the properties of a glass depend on the cooling rate? A computer simulation study of a Lennard-Jones system. J. Chem. Phys. 1996, 105, 4714-4728. [CrossRef]

53. Utz, M.; Debenedetti, P.G.; Stillinger, F.H. Atomistic simulation of aging and rejuvenation in glasses. Phys. Rev. Lett. 2000, 84, 1471-1474. [CrossRef] [PubMed] 